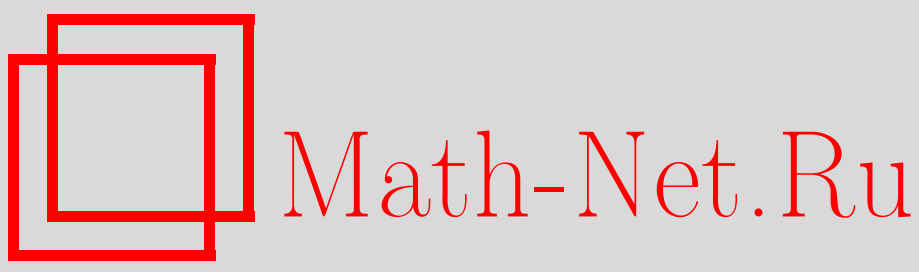

Н. Б. Енгибарян, Б. Н. Енгибарян, Интегральное уравнение свертки на полупрямой с вполне монотонным ядром, Матем. сб., 1996, том 187, номер 10, 53-72

DOI: https://doi.org/10.4213/sm164

Использование Общероссийского математического портала Math-Net.Ru подразумевает, что вы прочитали и согласны с пользовательским соглашением

http://www . mathnet.ru/rus/agreement

Параметры загрузки:

IP : 54.162 .27 .143

26 апреля 2023 г., 16:08:51 


\section{Интегральное уравнение свертки на полупрямой с вполне монотонным ядром}

Работа посвящена интегральному уравнению Винера-Хопфа

$$
f(x)=g(x)+\int_{0}^{\infty} K(x-t) f(t) d t, \quad(\boldsymbol{I}-\boldsymbol{K}) f=g
$$

и связанным с ним факторизационным задачам. Предполагается, что

$$
K( \pm x)=\int_{a}^{b} e^{-x p} d \sigma_{ \pm}(p), \quad \sigma_{ \pm}(p) \uparrow, \quad \mu \equiv \sum_{ \pm} \int_{a}^{b} \frac{1}{p} d \sigma_{ \pm}(p)<+\infty
$$

Доказьвается возможность вольтерровой факторизации в закритическом случае $($ (КС) $\mu>1$, если либо $K$ - четная функция, либо символ $1-\widehat{K}(s)$ обладает вещественным нулем. Указывается на обобщение резулттата на общий ЗКС. Доказывается разрешимость соответствующего уравнения (1) при $g \in L_{1}(0, \infty)$. Получается ряд других результатов по ЗКС и по случаю $\mu=1$. Излагаемьй подход существенньм образом опирается на метод специальной факторизации и на обобщенные уравнения В. А. Амбарцумяна.

Библиограф̆ия: 19 названий.

\section{Введение}

Рассмотрим интегральное уравнение

$$
f(x)=g(x)+\int_{0}^{\infty} K(x-t) f(t) d t
$$

где ядро (ядерная функция) $K$ представлено в виде суперпозиции экспонент

$$
K_{ \pm}(x) \equiv K( \pm x)=\int_{a}^{b} e^{-x p} d \sigma_{ \pm}(p), \quad x>0, \quad[a, b) \subset[0, \infty)
$$

Здесь $\sigma_{ \pm}$неубывающие, непрерывные слева функции на $[a, b)$, удовлетворяющие условию

$$
\mu \equiv \sum_{ \pm} \int_{a}^{b} \frac{1}{p} d \sigma_{ \pm}(p)=\int_{-\infty}^{\infty} K(x) d x<+\infty .
$$

Представление $(0.2)$ означает, что $K_{ \pm}$вполне монотонные на $(0, \infty)$ функции из $L_{1}(-\infty, \infty)$, т.е. они бесконечно дифференцируемы и $(-1)^{n} K_{ \pm}^{(n)}(x) \geqslant 0$, $n=0,1, \ldots$. 
Перепишем (0.1) в операторном виде

$$
(\boldsymbol{I}-\boldsymbol{K}) f=g
$$

где $\boldsymbol{I}$ - единичньй оператор, а $\boldsymbol{K}$ - интегральньй оператор Винера-Хопфа с ядром (0.2)

$$
(\boldsymbol{K} f)(x)=\int_{0}^{\infty} K(x-t) f(t) d t, \quad K \in L_{1}(-\infty, \infty)
$$

С точки зрения разрешимости уравнения $(0.1),(0.2)$ и свойств его решений качественно отличаются следующие три случая: диссипативный случай (ДС) $\mu<1$, консервативный случай $(\mathrm{KC}) \mu=1$ и закритический случай (ЗКС) $\mu>1$.

Пусть $E^{+} \equiv E[0, \infty)$ - одно из банаховых пространств $L_{p}^{+}(1 \leqslant p \leqslant+\infty$, $\left.L_{\infty}^{+} \equiv M^{+}\right), C_{u}^{+}, C_{0}^{+}$. Пространство $C_{u}^{+}$состоит из непрерьвных и ограниченных на $[0, \infty)$ функций, а $C_{0}^{+} \subset C_{u}^{+}$состоит из функций, стремящихся к 0 в $+\infty$. В ДС оператор $\boldsymbol{K}$ сжимающий и $\boldsymbol{I}-\boldsymbol{K}$ обратим в $E^{+}$. КС и ЗКС относятся к особым случаям уравнения (0.1). В $\mathrm{KC}$ и ЗКС $\boldsymbol{I}-\boldsymbol{K}$ необратим в $E^{+}$. Случай $\sigma_{ \pm}=\sigma$ называется симметрическим (CC). Тогда $K$ - четная функция.

Уравнения $(0.1),(0.2)$ в СС при $\mu \leqslant 1$ и $\sigma \in C_{\perp}[a, b)$ имеют важные применения в теории переноса излучения, в кинетической теории газов. Им посвящено огромное число теоретических и прикладных исследований (см. [1]-[5]). Несимметрический случай и случай, когда $\sigma_{ \pm} \notin C_{1}$ являются функциями локально ограниченной вариации, впервые изучены в работах [6], [7] (см. также [8]-[12]). Центральную роль в теории консервативных и диссипативных уравнений $(0.1),(0.2)$ играют уравнение В. А. Амбарцумяна и его обобщения (см. [1]-[4], [6], [7], [9]-[12]).

В 1982 году была опубликована большая работа М. Г. Крейна и Ю. Л. Шмульяна [12], в которой уравнение $(0.1),(0.2)$ было изучено с применением методов комплексного анализа. Достижением этой работы являются результаты по ЗКС. Что касается случаев $\mu \leqslant 1$, то основные результаты [12] (за исключением одного важного интегрального представления) являются новыми доказательствами или частными случаями соответствующих результатов работ [7], [9]. ${ }^{1}$ При этом наиболее важньй, КС, изучен при дополнительном ограничении конечности момента первого порядка ядра $K$.

Настоящая работа посвящена уравнению (0.1)-(0.3) и связанным с ним факторизационным задачам. Основное внимание уделяется ЗКС. Развиваемый подход достаточно конструктивен и примькает как к методам работ [7], [9]-[11], так и к [12]. Наряду с новыми результатами приводятся простые доказательства ряда результатов [12] по ЗKC.

Уравнение (0.1) в ЗКС не имеет прямых физических приложений. Однако оно может играть вспомогательную роль для решения задач генерации нейтронов в плоском слое и в шаре при докритическом или критическом режимах. Ряд таких задач описывается уравнением с симметрическим ядром, отличающимся от (0.1) тем, что верхний предел интегрирования $<+\infty$.

\footnotetext{
${ }^{1}$ Основные результаты препринта [9] содержатся в обзорной работе [10].
} 


\section{§ 1. Вспомогательные предложения}

Пусть $\Omega$ - класс интегральных операторов Винера-Хопфа вида (0.4) с произвольным $K \in L_{1}(-\infty, \infty)$. Оператор $\boldsymbol{K} \in \Omega$ действует в $E^{+}$, причем (см. [13]-[15])

$$
\|\boldsymbol{K}\|_{E^{+}} \leqslant \mu \equiv \int_{-\infty}^{\infty}|K(x)| d x .
$$

В случае $K \geqslant 0$ неравенство обрашается в равенство.

Пусть $\Omega^{+}, \Omega^{-} \subset \Omega$ - алгебры нижних и верхних вольтерровых операторов: $\boldsymbol{V}_{ \pm} \in \Omega^{ \pm}$, если

$$
\boldsymbol{V}_{+} f(x)=\int_{0}^{x} V_{+}(x-t) f(t) d t, \quad \boldsymbol{V}_{-} f(x)=\int_{x}^{\infty} V_{-}(t-x) f(t) d t
$$

где $V_{ \pm} \in L_{1}^{+}$. Если $\boldsymbol{V}_{ \pm} \in \Omega^{ \pm}$, то $\boldsymbol{V}_{-} \boldsymbol{V}_{+} \in \Omega$ (см. [7]).

Резольвентные функции $\Phi_{ \pm}$операторов $\boldsymbol{V}_{ \pm}$определяются из следуюших уравнений типа восстановления

$$
\Phi_{ \pm}(x)=V_{ \pm}(x)+\int_{0}^{x} V_{ \pm}(x-t) \Phi_{ \pm}(t) d t .
$$

Эти уравнения всегда однозначно разрешимы в пространстве $L_{1}^{\text {loc }}$ локально интегрируемых на $[0, \infty)$ функций. Высказывание $\Phi_{ \pm} \in L_{1}^{+}$эквивалентно обратимости $\boldsymbol{I}-\boldsymbol{V}_{ \pm}$в $E^{+}:\left(\boldsymbol{I}-\boldsymbol{V}_{ \pm}\right)^{-1}=\boldsymbol{I}+\boldsymbol{\Phi}_{ \pm}$, где $\boldsymbol{\Phi}_{ \pm} \in \Omega^{ \pm}$- операторы с ядрами $\Phi_{ \pm}$. Если $\Phi_{+} \notin L_{1}^{+}$или $\Phi_{-} \notin L_{1}^{+}$, то $\boldsymbol{\Phi}_{ \pm}$имеют смысл как операторы, действующие в подходящим образом выбранных пространствах.

Пусть $1-\widehat{K}(s)$ - символ оператора $\boldsymbol{I}-\boldsymbol{K}, \boldsymbol{K} \in \Omega$, где $\widehat{K}$ - преобразование Фурье от $K$

$$
\widehat{K}(s)=\int_{-\infty}^{\infty} K(x) e^{i s x} d x .
$$

Для обратимости $\boldsymbol{I}-\boldsymbol{K}$ в $E^{+}$, хотя бы односторонней, необходимо, чтобы (см. [13], [14])

$$
1-\widehat{K}(s) \neq 0, \quad \operatorname{Im} s=0 .
$$

При выполнении (1.4) $\boldsymbol{I}-\boldsymbol{K}$ будет обратим, если

$$
\varkappa=\operatorname{ind}(1-\widehat{K})=-\frac{1}{2 \pi} \int_{-\infty}^{\infty} d \arg [1-\widehat{K}(s)]=0 .
$$

Если индекс $\varkappa<0$, то $\boldsymbol{I}-\boldsymbol{K}$ обратим только слева, а если $\varkappa>0$ - только справа.

Пусть произведение $\left(\boldsymbol{I}-\boldsymbol{K}_{1}\right) \cdots\left(\boldsymbol{I}-\boldsymbol{K}_{n}\right)$, где $\boldsymbol{K}_{m} \in \Omega, m=\overline{1, n}$, является оператором такого же вида, т.е. справедливо равенство

$$
\boldsymbol{I}-\boldsymbol{K}=\left(\boldsymbol{I}-\boldsymbol{K}_{1}\right) \cdots\left(\boldsymbol{I}-\boldsymbol{K}_{n}\right), \quad \boldsymbol{K} \in \Omega,
$$

тогда в (1.6) можно перейти к символам (см. [16])

$$
1-\widehat{K}(s)=\left(1-\widehat{K}_{1}(s)\right) \cdots\left(1-\widehat{K}_{n}(s)\right) .
$$


Равенства (1.6) и (1.7) эквивалентны.

Рассмотрим класс $\Omega_{0} \subset \Omega$ операторов $\boldsymbol{T}$ из $\Omega$, ядра которых допускают представление

$$
T_{ \pm}(x) \equiv T( \pm x)=\int_{a}^{b} e^{-x p} d \rho_{ \pm}(p), \quad x>0, \quad a \geqslant 0 .
$$

Здесь $\rho_{ \pm}-$комплекснозначные функции локально ограниченной вариации на $[a, b)$, удовлетворяющие условию

$$
\lambda \equiv \sum_{ \pm} \int_{a}^{b} \frac{1}{p}\left|d \rho_{ \pm}(p)\right|<+\infty .
$$

Имеем

$$
\|\boldsymbol{T}\|_{E^{+}} \leqslant\|\boldsymbol{T}\|_{L_{1}(-\infty, \infty)} \leqslant \lambda .
$$

Введем также классы $\Omega_{0}^{ \pm}=\Omega_{0} \cap \Omega^{ \pm}$.

С уравнением (0.1) с ядром $K=T$ вида (1.8) ассоциируется следуюшая система нелинейных функциональных уравнений, которая является обобшением известного уравнения Амбарцумяна в теории переноса излучения (см. [1]-[4], [6]-[12])

$$
\varphi_{ \pm}(p)=1+\varphi_{ \pm}(p) \int_{a}^{b} \frac{\varphi_{\mp}(q)}{p+q} d \rho_{\mp}(q) .
$$

Пусть $\mathscr{L}^{+}$и $\mathscr{L}^{-}$- банаховы пространства с нормами

$$
\left\|\varphi_{ \pm}\right\|_{\mathscr{L}^{ \pm}}=\int_{a}^{b} \frac{1}{p}\left|\varphi_{ \pm}(p)\right|\left|d \rho_{ \pm}(p)\right| .
$$

Если (1.11) обладает решением $\left(\varphi_{+}, \varphi_{-}\right) \in \mathscr{L}^{+} \times \mathscr{L}^{-}$, то имеет место факторизация (см. [7])

$$
\boldsymbol{I}-\boldsymbol{T}=\left(\boldsymbol{I}-\boldsymbol{V}_{-}\right)\left(\boldsymbol{I}-\boldsymbol{V}_{+}\right),
$$

где $\boldsymbol{V}_{ \pm} \in \Omega_{0}^{ \pm}$- операторы вида (1.2), с ядрами

$$
V_{ \pm}(x)=\int_{a}^{b} e^{-x p} \varphi_{ \pm}(p) d \rho_{ \pm}(p), \quad x>0 .
$$

Каноническим (или главньм) решением (КР) уравнения Амбарцумяна (УА) (1.11) называется предел в $\mathscr{L}^{+} \times \mathscr{L}^{-}$итераций $\left(\varphi_{n}^{+}, \varphi_{n}^{-}\right)$:

$$
\varphi_{n+1}^{ \pm}(p)=1+\varphi_{n}^{ \pm}(p) \int_{a}^{b} \frac{\varphi_{n}^{\mp}(q)}{p+q} d \rho_{\mp}(q), \quad \varphi_{0}^{ \pm}=0, \quad n=0,1, \ldots
$$

При $\lambda \leqslant 1$ существует $\mathrm{KP}\left(\varphi_{+}, \varphi_{-}\right)$, которое обладает следующими свойствами:

$$
\gamma_{ \pm} \equiv\left\|\varphi_{ \pm}\right\|_{\mathscr{L}^{ \pm}} \leqslant \lambda, \quad \gamma_{+}+\gamma_{-} \leqslant 2(1-\sqrt{1-\lambda}), \quad\left\|\varphi_{n}^{ \pm}-\varphi_{ \pm}\right\|_{\mathscr{L}^{ \pm}} \leqslant \lambda^{n+1} .
$$

Функции $\varphi_{ \pm}$непрерывны на $(a, b), \varphi_{ \pm}(+\infty)=1$. Они голоморфно продолжаются на комплексную плоскость $\mathbb{C}$ с разрезом $[-b,-a]$ по формуле

$$
\varphi_{ \pm}(p)=\left(1-\int_{a}^{b} \frac{\varphi_{\mp}(q)}{p+q} d \rho_{\mp}(q)\right)^{-1} .
$$


Если функции $\rho_{ \pm}$вешественные, то таковыми являются и $\varphi_{ \pm}$. Если $\rho_{ \pm}$неубываюшие функции, т.е. меры $d \rho_{ \pm}$неотрицательны, то

$$
0 \leqslant \varphi_{ \pm}(p) \downarrow \text { по } p \text { на }(0, \infty) \text { и }\left(1-\gamma_{-}\right)\left(1-\gamma_{+}\right)=1-\lambda \text {. }
$$

В симметрическом случае, когда $\rho_{ \pm}=\rho$ и $\mathscr{L}^{ \pm}=\mathscr{L}$, имеем $\varphi_{ \pm}=\varphi$, где $\varphi$ является КР УА

$$
\varphi(p)=1+\varphi(p) \int_{a}^{b} \frac{\varphi(q)}{p+q} d \rho(q) .
$$

$\mathrm{B} \mathrm{CC} \varphi_{n}^{ \pm}=\varphi_{n}$,

$$
\gamma \equiv\|\varphi\|_{\mathscr{L}} \leqslant 1-\sqrt{1-\lambda}, \quad\left\|\varphi-\varphi_{n}\right\|_{\mathscr{L}} \leqslant \min \left\{\frac{2}{n},(1-\sqrt{1-\lambda})^{n+1}\right\} .
$$

При $d \rho \geqslant 0$ первое из неравенств (1.19) обрашается в равенство.

Рассмотрим моменты функций $T$ и $V_{ \pm}$. Пусть $f \in L_{1}^{+}$. Обозначим через $m_{p}(f)$, где $p \geqslant 0$, момент $p$-го порядка функции $f$

$$
m_{p}(f)=\int_{0}^{\infty} x^{p} f(x) d x .
$$

Если не будет оговорено противное, то будем считать, что этот интеграл абсолютно сходится. Если же $f \geqslant 0$, то рассматриваемым моментам будем приписывать также значение $+\infty$. Обозначим

$$
\begin{aligned}
& \nu_{n}^{ \pm}=m_{n}\left(T_{ \pm}\right)=n ! \int_{a}^{b} \frac{1}{p^{n+1}} d \rho_{ \pm}(p), \\
& d_{n}^{ \pm}=m_{n}\left(V_{ \pm}\right)=n ! \int_{a}^{b} \frac{1}{p^{n+1}} \varphi_{ \pm}(p) d \rho_{ \pm}(p) .
\end{aligned}
$$

Сформулируем некоторые факты из [9], [10] по моментам $V_{ \pm}$в КС. Пусть выполняются условия консервативности $T \geqslant 0$ и $\lambda=1$. Из равенства (1.17) следует, что тогда хотя бы одно из чисел $\gamma_{ \pm}$равно 1. В СС $\gamma_{ \pm}=\gamma=1$. Что касается несимметрического случая, то справедливы следующие утверждения.

Пусть хотя бы одно из чисел $\nu_{1}^{ \pm}$конечно. Обозначим $\nu_{1}=\nu_{1}^{+}-\nu_{1}^{-}$, $-\infty \leqslant \nu_{1} \leqslant+\infty$. Тогда
a) $\nu_{1}=0 \Longleftrightarrow \gamma_{ \pm}=1$;
б) $\nu_{1}>0 \Longleftrightarrow \gamma_{+}=1, \gamma_{-}<1$;
в) $\nu_{1}<0 \Longleftrightarrow \gamma_{+}<1, \gamma_{-}=1$.

Рассмотрим симметрический КС. Пусть $\nu_{2}^{ \pm}=\frac{1}{2} \nu_{2}$. Тогда имеет место равенство

$$
\alpha_{1}^{ \pm}=\alpha_{1}=\sqrt{\frac{1}{2} \nu_{2}} \quad(\leqslant+\infty) .
$$

Изучим вопрос о решении уравнений (1.3) относительно резольвентных функций $\Phi_{ \pm}$в случае, когда $V_{ \pm}$задаются в $(1.13)$, где $d \rho_{ \pm} \geqslant 0$ и $\gamma_{ \pm} \leqslant 1$. Согласно результатам [12] существуют неотрицательные меры $d \eta_{ \pm}$такие, что

$$
\Phi_{ \pm}(x)=\int_{0}^{\infty} e^{-x p} d \eta_{ \pm}(p)
$$


В работах [17], [18] найден эффективньй способ построения представления (1.22) и получены дополнительные свойства этих мер.

\section{§2. Вопрос обратимости $I-K$}

Рассмотрим оператор $\boldsymbol{K} \in \Omega_{0}$ с ядром (0.2). Символ оператора $\boldsymbol{I}-\boldsymbol{K}$ имеет вид

$$
1-\widehat{K}(s)=1-\int_{a}^{b} \frac{1}{p-i s} d \sigma_{+}(p)-\int_{a}^{b} \frac{1}{p+i s} d \sigma_{-}(p) .
$$

Функция $1-\widehat{K}$ голоморфно продолжается с вешественной оси $\operatorname{Im} s=0$ в $\mathbb{C}$ с двумя разрезами вдоль мнимой оси $[i a, i b]$ и $[-i b, i a]$.

Обозначим $\sigma=\frac{1}{2}\left(\sigma_{+}+\sigma_{-}\right)$и $\xi=\frac{1}{2}\left(\sigma_{+}-\sigma_{-}\right)$. Из (2.1) имеем

$$
1-\widehat{K}(s)=1-\int_{a}^{b} \frac{2 p}{p^{2}+s^{2}} d \sigma(p)-2 i s \int_{a}^{b} \frac{1}{p^{2}+s^{2}} d \xi(p) .
$$

Введем функции

$$
f_{1}(\gamma)=1-\int_{a}^{b} \frac{2 p}{p^{2}+\gamma} d \sigma(p), \quad f_{2}(s)=2 \int_{a}^{b} \frac{1}{p^{2}+s^{2}} d \xi(p)
$$

Имеем

$$
1-\widehat{K}(s)=f_{1}\left(s^{2}\right)-i s f_{2}(s) .
$$

В СС, когда $\sigma_{ \pm}=\sigma$, получаем $\xi=0, f_{2}=0$.

Проверим выполнение условий (1.4), (1.5) обратимости $\boldsymbol{I}-\boldsymbol{K}$ в $E^{+}$. В ДС оба условия выполняются. В КС имеем $1-\widehat{K}(0)=0$, т.е. нарушается условие (1.4). Покажем, что и в ЗКС нарушается одно из условий $(1.4),(1.5)$. Функция $f_{1}(\gamma)$ на $[0, \infty)$ обладает следуюшими очевидньми свойствами:

$$
f_{1}(0)=1-\mu<0, \quad f_{1} \in C_{u}^{+}, \quad f_{1}(\gamma) \uparrow \text { по } \gamma\left(\text { строго) и } f_{1}(+\infty)=0 .\right.
$$

Из отмеченных свойств $f_{1}$ следует сушествование единственного корня $\gamma>0$ на $[0, \infty)$. Пусть $\omega=\sqrt{\gamma}$. Тогда

$$
f_{1}\left(\omega^{2}\right)=0 .
$$

Возможны следующие случаи:

a) $f_{2}(\omega)=0$

б) $f_{2}(\omega)>0$

в) $f_{2}(\omega)<0$.

В первом случае имеем $1-\widehat{K}( \pm \omega)=0$, т.е. условие (1.4) нарушается в точках $s= \pm \omega$. Так обстоит дело, в частности, в симметрическом ЗКС. Если же $f_{2}(\omega) \neq 0$, то $1-\widehat{K}$ не имеет вешественных нулей, т.е. условие (1.4) выполняется.

Лемма 1.1. Если $f_{2}(\omega)>0$, mo $\varkappa=-1$. Если $f_{2}(\omega)<0$, mo $\varkappa=+1$. 
ДокАЗАТЕЛЬСтво. Пусть $G^{ \pm}$следующие подмножества компактифицированной вешественной оси $\overline{\mathbb{R}}=[-\infty, \infty]$ :

$$
G^{+}=\left\{s>\omega ; s f_{2}(s)=0\right\}, \quad G^{-}=\left\{s<\omega ; s f_{2}(s)=0\right\} .
$$

Эти множества непусты, так как $\{0\} \in G^{-}$и $\pm \infty \in G^{+}$. В силу непрерывности $f_{2}$ на $\overline{\mathbb{R}}$ и $f_{2}(\omega) \neq 0$ множества $G^{ \pm}$замкнуты. Обозначим $s_{1}=\max G^{-}(<\omega)$ и $s_{2}=\min G^{+}(>\omega)$. Представим значение $(1.5)$ индекса $\varkappa$ в виде суммы пяти слагаемых: $\varkappa=\varkappa_{1}+\cdots+\varkappa_{5}$, где

$$
\begin{array}{cc}
\varkappa_{1}=-\int_{-\infty}^{-s_{2}} \frac{1}{2 \pi} d \arg [1-\widehat{K}(s)], & \varkappa_{2}=-\int_{-s_{2}}^{-s_{1}} \frac{1}{2 \pi} d \arg [1-\widehat{K}(s)], \\
\varkappa_{3}=-\int_{-s_{1}}^{s_{1}} \frac{1}{2 \pi} d \arg [1-\widehat{K}(s)], \quad \varkappa_{4}=-\int_{s_{1}}^{s_{2}} \frac{1}{2 \pi} d \arg [1-\widehat{K}(s)], \\
\varkappa_{5}=-\int_{s_{2}}^{\infty} \frac{1}{2 \pi} d \arg [1-\widehat{K}(s)] .
\end{array}
$$

С учетом монотонности функции $f_{2}$ нетрудно убедиться, что $\varkappa_{1}=\varkappa_{3}=\varkappa_{5}=0$. При $f_{2}(\omega)>0$ получаем $\varkappa_{2}=\varkappa_{4}=-\frac{1}{2}$, т.е. $\varkappa=-1$. Если же $f_{2}(\omega)<0$, то $\varkappa_{2}=\varkappa_{4}=\frac{1}{2}$. Лемма доказана.

Итак, в ЗКС при $f_{2}(\omega)=0$ (в частности, в СС) оператор $\boldsymbol{I}-\boldsymbol{K}$ необратим ни справа, ни слева. При $f_{2}(\omega)<0, \boldsymbol{I}-\boldsymbol{K}$ обратим только справа, а при $f_{2}(\omega)>0-$ только слева.

Несколько слов о комплексных корнях символа $1-\widehat{K}$. Из (2.1) имеем (черточкой сверху отмечается переход к сопряженной комплексной величине)

$$
\overline{1-\widehat{K}(s)}=1-\int_{a}^{b} \frac{d \sigma_{+}(p)}{p+i \bar{s}}-\int_{a}^{b} \frac{d \sigma_{-}(p)}{p-i \bar{s}}=1-\widehat{K}(-\bar{s}) .
$$

Отсюда видно, что если число $s=s_{0}$ является нулем символа $1-\widehat{K}$, то $-\bar{s}_{0}$ также является его нулем.

В симметрическом ДС символ может иметь два чисто мнимых нуля $\pm i \delta$, где $\delta>0$ определяется из характеристического уравнения хорошо известного в теории переноса излучения

$$
\int_{a}^{b} \frac{2 p}{p^{2}-\delta^{2}} d \sigma(p)=1
$$

\section{§3. Подготовительная задача факторизации}

3.1. Рассмотрим оператор $\boldsymbol{K} \in \Omega_{0}$ в предположении, что $1-\widehat{K}$ обладает вещественными нулями $\pm \omega$, т.е. $f_{2}(\omega)=0$. В настоящем и следующем параграфах мы будем заниматься решением основных факторизационных задач для оператоpa $\boldsymbol{I}-\boldsymbol{K}$ в ЗКС. Эти задачи представляют как самостоятельный интерес, так и важны с точки зрения применения к уравнению (0.1)-(0.3).

Обозначим через $\boldsymbol{U}_{ \pm} \in \Omega_{0}$ следуюшие простейшие вольтерровы операторы, где $\beta>0, \alpha \in \mathbb{C}$,

$$
\boldsymbol{U}_{+} f(x)=\alpha \int_{0}^{x} e^{-\beta(x-t)} f(t) d t, \quad \boldsymbol{U}_{-} f(x)=\alpha \int_{x}^{\infty} e^{-\beta(t-x)} f(t) d t .
$$


Поставим задачу о построении факторизации

$$
\boldsymbol{I}-\boldsymbol{K}=\left(\boldsymbol{I}-\boldsymbol{U}_{-}\right)(\boldsymbol{I}-\boldsymbol{T})\left(\boldsymbol{I}-\boldsymbol{U}_{+}\right),
$$

$\boldsymbol{T} \in \Omega_{0}$ оператор с ядром вида $(1.8)$, где $d \rho_{ \pm}(p)=h(p) d \sigma_{ \pm}(p), h-$ непрерывная на $(a, b)$ функция, удовлетворяющая условию

$$
\lambda \equiv 2 \int_{a}^{b}|h(p)| \frac{1}{p} d \sigma(p)<1
$$

Мы будем стремиться, по возможности, обеспечить выполнение дополнительного условия

$$
h(p) \geqslant 0, \quad p \in(a, b),
$$

что влечет за собой неотрицательность мер $h(p) d \sigma_{ \pm}(p)$.

Соответствующие выкладки проще всего выполнить, используя технику преобразования Фурье, опираясь при этом на эквивалентность равенств (1.6) и (1.7). Символами операторов $\boldsymbol{I}-\boldsymbol{U}_{ \pm}$и $\boldsymbol{I}-\boldsymbol{T}$ служат функции

$$
\begin{gathered}
1-\widehat{U}_{ \pm}(s)=\frac{\beta-\alpha \mp i s}{\beta \mp i s} \\
1-\widehat{T}(s)=1-\int_{a}^{b} \frac{2 p h(p)}{p^{2}+s^{2}} d \sigma(p)-2 i s \int_{a}^{b} \frac{h(p)}{p^{2}+s^{2}} d \xi(p) .
\end{gathered}
$$

Переходя в (3.1) к символам, получаем

$$
1-\widehat{K}(s)=\frac{(\beta-\alpha)^{2}+s^{2}}{\beta^{2}+s^{2}}\left(1-\int_{a}^{b} \frac{2 p h(p)}{p^{2}+s^{2}} d \sigma(p)-2 i s \int_{a}^{b} \frac{h(p)}{p^{2}+s^{2}} d \xi(p)\right) .
$$

Ниже мы будем заниматься построением разложения (3.5) как при $\beta>0$, так и при $\beta=0$. Считаем, что $\omega \geqslant 0$.

Используя выражение $(2.2)$ для $1-\widehat{K}$ и равенства $1-\widehat{K}( \pm \omega)=0$, имеем

$$
\begin{aligned}
1-\widehat{K}(s)=\int_{a}^{b}\left(\frac{1}{p^{2}+\omega^{2}}\right. & \left.-\frac{1}{p^{2}+s^{2}}\right) 2 p d \sigma(p) \\
& +2 i s \int_{a}^{b}\left(\frac{1}{p^{2}+\omega^{2}}-\frac{1}{p^{2}+s^{2}}\right) d \xi(p) \\
= & \frac{s^{2}-\omega^{2}}{s^{2}+\beta^{2}}\left(\int_{a}^{b} \frac{\left(s^{2}+\beta^{2}\right) 2 p}{\left(p^{2}+s^{2}\right)\left(p^{2}+\omega^{2}\right)} d \sigma(p)\right. \\
& \left.+2 i s \int_{a}^{b} \frac{\left(s^{2}+\beta^{2}\right)}{\left(p^{2}+s^{2}\right)\left(p^{2}+\omega^{2}\right)} d \xi(p)\right)
\end{aligned}
$$

откуда

$$
1-\widehat{K}(s)=\frac{s^{2}-\omega^{2}}{s^{2}+\beta^{2}}\left(1-\int_{a}^{b} \frac{2 p h(p)}{p^{2}+s^{2}} d \sigma(p)-2 i s \int_{a}^{b} \frac{h(p)}{p^{2}+s^{2}} d \xi(p)\right),
$$

где $\beta \geqslant 0$ произвольное неотрицательное число, $\omega \geqslant 0$,

$$
h(p)=\frac{p^{2}-\beta^{2}}{p^{2}+\omega^{2}} .
$$


При $\beta>0$ из (3.5) и (3.6) приходим к разложению (3.1), где $\alpha=\beta-i \omega$, a $h$ определяется из (3.7), т.е.

$$
T_{ \pm}(x) \equiv T( \pm x)=\int_{a}^{b} e^{-x p} \frac{p^{2}-\beta^{2}}{p^{2}+\omega^{2}} d \sigma_{ \pm}(p), \quad x>0 .
$$

Обратим внимание на то обстоятельство, что при $\omega>0$ ядро $T$, определенное согласно (3.8), принадлежит $L_{1}(-\infty, \infty)$ как в случае $\beta>0$, так и в случае $\beta=0$. Сказанное следует из неравенства $|h(p)| \leqslant 1+\beta^{2} / \omega^{2}$. Поэтому $\lambda \leqslant\left(1+\beta^{2} / \omega^{2}\right) \mu<+\infty$ и $\boldsymbol{T} \in \Omega_{0}$. Очевидно также, что при $\beta=0$ ядро $T$ является консервативным.

$\mathrm{B} \mathrm{KC}$, когда $\omega=0$, имеем

$$
\boldsymbol{T}_{ \pm}(x)=\int_{a}^{b} e^{-x p}\left(1-\frac{\beta^{2}}{p^{2}}\right) d \sigma_{ \pm}(p) .
$$

Поэтому при $\beta>0$ для того, чтобы $\boldsymbol{T} \in \Omega_{0}$ необходимо и достаточно, чтобы исходное ядро $K$ обладало конечным моментом второго порядка $\nu_{2}^{ \pm} \equiv m_{2}\left(K_{ \pm}\right)<+\infty$. Тогда $\lambda \leqslant \mu+\beta^{2}\left(\nu_{2}^{+}+\nu_{2}^{-}\right)$.

3.2. Рассмотрим вопрос выбора значений свободного параметра $\beta>0$, при которых выполняется неравенство (3.3). Более того, мы будем минимизировать величину $\lambda=\lambda(\beta)$ в случае непрерывной функции $\sigma$. Начнем со случая $a>0$. Тогда функции $K_{ \pm}$экспоненциально убывают на $\infty$ и $1-\widehat{K}(s)$ голоморфна в полосе $|\operatorname{Im} s|<a$. При $\beta \leqslant a$ функция $h$ неотрицательна на $(a, b)$ и

$$
\lambda(\beta)=\int_{a}^{b}\left(p^{2}-\beta^{2}\right) \frac{2}{p\left(p^{2}+\omega^{2}\right)} d \sigma(p)=1-\beta^{2} \int_{a}^{b} \frac{2}{p\left(p^{2}+\omega^{2}\right)} d \sigma(p) .
$$

Имеем $\lambda(0)=1$, поэтому $\lambda(\beta)<1$ при $0<\beta \leqslant a$ и $\lambda_{\min }=\lambda(a)$ на $[0, a]$. Если точка $a$ является точкой роста функции $\sigma$ (без ограничения общности можно считать, что это так), то $\beta=a$ является наибольшим значением $\beta$, при котором меры $h(p) d \sigma_{ \pm}(p)$ неотрицательны.

Пусть теперь $a=0$, в $\mathrm{KC} \nu_{2}^{ \pm}<+\infty$. Покажем, что при достаточно малых значениях $\beta>0$ выполняется неравенство (3.2). Данное неравенство принимает вид

$$
\int_{0}^{\beta}\left(\beta^{2}-p^{2}\right) d \sigma_{1}(p)+\int_{\beta}^{b}\left(p^{2}-\beta^{2}\right) d \sigma_{1}(p)<1
$$

где

$$
d \sigma_{1}(p)=\frac{2}{p\left(p^{2}+\omega^{2}\right)} d \sigma(p), \quad \sigma_{1}(p)=\int_{0}^{p} \frac{2}{q\left(q^{2}+\omega^{2}\right)} d \sigma(q) .
$$

С учетом $\lambda(0)=1,(3.9)$ можно привести к виду

$$
2 \beta^{2} \int_{0}^{\beta} d \sigma_{1}(p)<\beta^{2} \int_{0}^{b} d \sigma_{1}(p)+2 \int_{0}^{\beta} p^{2} d \sigma_{1}(p) .
$$

Для выполнения этого неравенства достаточно, чтобы

$$
\int_{0}^{\beta} d \sigma_{1}(p)<\frac{1}{2} \int_{0}^{b} d \sigma_{1}(p)
$$


Правая часть неравенства положительна, а левая часть стремится к 0 при $\beta \rightarrow 0+$ (в силу сходимости собственного или несобственного интеграла $\left.\int_{0}^{b} d \sigma_{1}(p)\right)$. Отсюда следует (3.11) при достаточно малых значениях $\beta>0$. Такие значения мы назовем допустимыми значениями $\beta$.

Рассмотрим вопрос минимизации $\lambda(\beta)$ при $a=0$, в случае непрерывной функции $\sigma$. Пусть $\sigma \in C(0, b)$. Можно показать, что $\lambda^{\prime}(\beta)=2 \beta \psi(\beta)$, где $\psi(\beta)=\int_{0}^{\beta} d \sigma_{1}(p)-\int_{\beta}^{b} d \sigma_{1}(p)$. В случае $\sigma \in C_{1}(0, b)$ эта формула очевидна. При $\sigma \in C(0, b)$ ее вывод связан с небольшими техническими трудностями. Из непрерывности $\sigma$ и сходимости интегралов (0.3) следует непрерывность на $[0, b)$ функции $\sigma_{1}$, определенной согласно (3.10). Имеем $\psi(\beta)=2 \sigma_{1}(\beta)-\sigma_{1}(b)$. Поэтому $\psi(\beta)$ - непрерывная неубывающая на $[a, b], b \leqslant+\infty$, функция, причем $\psi(0)=-\sigma_{1}(b)<0$ и $\psi(b)=\sigma_{1}(b)>0$. Следовательно, сушествует точка $\beta=\beta_{0}>0$ (или интервал значений $\beta$ ), в которой $\psi\left(\beta_{0}\right)=0$, т.е.

$$
\int_{0}^{\beta_{0}} \frac{1}{p\left(p^{2}+\omega^{2}\right)} d \sigma(p)=\frac{1}{2} \int_{0}^{b} \frac{1}{p\left(p^{2}+\omega^{2}\right)} d \sigma(p)
$$

Точка $\beta_{0}$ является точкой абсолютного минимума функции $\lambda(\beta)$.

Объединяя результаты настоящего параграфа мы приходим к следующей теореме.

ТЕОРемА 3.1. Пусть выполняется одно из следующих условий:

а) в $3 K C, \mu>1$, либо $K$ - четная функция, либо символ $1-\widehat{K}(s)$ обладает вешественным нулем $\omega>0$;

б) в $K C, \mu=1$, ядро $K$ обладает конечным моментом второго порядка.

Тогда существует факторизация (3.1), (3.2), где $\beta>0$ удовлетворяет условию (3.11); в случае $\sigma \in C(0, b)$ число $\beta>0$ мохет быть определено из условия минимума $\lambda(\beta)$ (3.12); если $а>0$, то при $\beta \leqslant$ в выполняются условия (3.3) и (3.2) и функиии $T_{ \pm}$вполне монотонны на $(0, \infty)$. Число $\alpha=\beta-i \omega$, әде $\omega=0$ в $K C$, а в ЗКС $\omega>0$, является единственным положстельным нулем символа $1-\widehat{K}$.

\section{§4. Основные задачи факторизации}

4.1. Дальнейшие результаты по факторизации оператора $\boldsymbol{I}-\boldsymbol{K}$ опираются на применение уравнения Амбарцумяна (1.11). В силу неравенства (3.2) оператор $\boldsymbol{I}-\boldsymbol{T}$, участвующий в разложении (3.1), допускает факторизацию (1.12). Ядра $V_{ \pm}$вольтерровых факторов $\boldsymbol{V}_{ \pm}$выражаются через функции Амбарцумяна $\varphi_{ \pm}$ по формулам (1.13). Пусть $\varphi_{ \pm}(p)=\varphi_{ \pm}(p, \beta), \beta \geqslant 0$, является КР УА (1.11), соответству ющего мерам $d \rho_{ \pm}$вида

$$
d \rho_{ \pm}(p)=h(p) d \sigma_{ \pm}(p)=\frac{p^{2}-\beta^{2}}{p^{2}+\omega^{2}} d \sigma_{ \pm}(p)
$$

Ниже мы установим некоторые дополнительњые свойства функций $\varphi_{ \pm}(p)$. Сначала приведем одно простое и полезное свойство этих функций, которое раньше 
не было отмечено. Из (1.16) и неравенств (1.15) непосредственно следует, что в случае вещественных мер $d \rho_{ \pm}$имеют место неравенства

$$
\varphi_{ \pm}(p)>0, \quad p \in(0, \infty)
$$

Положительность $\varphi_{ \pm}$независимо от знакопеременности мер $d \rho_{ \pm}$играет важную роль в ряде теоретических и прикладных вопросов, в том числе - в вопросе построения резольвентных функций $\Phi_{ \pm}$из $(1.3)$ в виде суперпозиций экспонент типа (1.22).

Лемма 4.1. KP $\left(\varphi_{+}, \varphi_{-}\right)$УA (1.11), (4.1) nрu $\mu>1 u \beta>0$ обладает свойством

$$
\int_{a}^{b} \frac{\varphi_{ \pm}(p, \beta)(p+\beta)}{p^{2}+\omega^{2}} d \sigma_{ \pm}(p)=1
$$

Это свойство означает, что символь $1-\widehat{V}_{+}$и $1-\widehat{V}_{-}$операторов $\boldsymbol{I}-\boldsymbol{V}_{ \pm}$

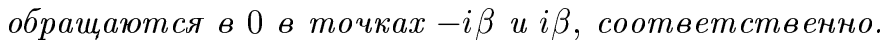

ДокАЗАтЕльство. Полагая в выражении (3.4) для $1-\widehat{T}(s) s= \pm i \beta$, с учетом $(3.7)$ и $1-\widehat{K}(\omega)=0$ получаем

$$
1-\widehat{T}( \pm i \beta)=1-\int_{a}^{b} \frac{2 p}{p^{2}+\omega^{2}} d \sigma(p) \pm 2 \beta \int_{a}^{b} \frac{1}{p^{2}+\omega^{2}} d \xi(p)=0
$$

Из (1.12) согласно формуле (1.7) имеем

$$
1-\widehat{T}(s)=\left(1-\widehat{V}_{-}(s)\right)\left(1-\widehat{V}_{+}(s)\right)
$$

где

$$
\widehat{V}_{ \pm}(s)=\int_{a}^{b} \frac{\varphi_{ \pm}(p, \beta)\left(p^{2}-\beta^{2}\right)}{(p \mp i s)\left(p^{2}+\omega^{2}\right)} d \sigma_{ \pm}(p) .
$$

Из неравенств (1.15) следует, что $1-\widehat{V}_{-}$и $1-\widehat{V}_{+}$при $\lambda<1$ не обращаются в 0 в “своих" (нижней и верхней) замкнутых полуплоскостях $\overline{\mathbb{C}}^{-}$и $\overline{\mathbb{C}}^{+}$, соответственно. Поэтому из $(4.4)$ и $(4.5)$ следует $1-\widehat{V}_{\mp}( \pm i \beta)=0$. Лемма доказана.

Нами доказана

Теорема 4.1. В условиях теоремы 3.1 имеет место факторизачия

$$
\boldsymbol{I}-\boldsymbol{K}=\left(\boldsymbol{I}-\boldsymbol{U}_{-}\right)\left(\boldsymbol{I}-\boldsymbol{V}_{-}\right)\left(\boldsymbol{I}-\boldsymbol{V}_{+}\right)\left(\boldsymbol{I}-\boldsymbol{U}_{+}\right),
$$

где $\boldsymbol{U}_{ \pm}$определяются, как в $n .3 .1 ; \boldsymbol{V}_{ \pm} \in \Omega_{0}^{ \pm}-$операторь вида (1.2) с ядрами (1.13), причем $\varphi_{ \pm}(p)=\varphi_{ \pm}(p, \beta)$ является КР УА (1.11), (4.1) и обладает свойствами (1.15), (4.2), (4.3). В СС имеем $\varphi_{ \pm}=\varphi$ и $V_{ \pm}=V$. 
4.2. Используем (4.7) для построения других разложений оператора $\boldsymbol{I}-\boldsymbol{K}$.

Так как классы $\Omega^{+}$и $\Omega^{-}$вольтерровых операторов являются коммутативньми алгебрами, то разложение (4.7) можно переписать в виде

$$
\boldsymbol{I}-\boldsymbol{K}=\left(\boldsymbol{I}-\boldsymbol{V}_{-}\right)(\boldsymbol{I}-\boldsymbol{\Lambda})\left(\boldsymbol{I}-\boldsymbol{V}_{+}\right),
$$

где $\boldsymbol{I}-\boldsymbol{\Lambda}=\left(\boldsymbol{I}-\boldsymbol{U}_{-}\right)\left(\boldsymbol{I}-\boldsymbol{U}_{+}\right)$. Простые вычисления приводят нас к следуюшему выражению для оператора $\boldsymbol{\Lambda}$

$$
\begin{gathered}
\Lambda f(x)=c_{0} \int_{0}^{\infty} e^{-\beta|x-t|} f(t) d t \\
c_{0}=\frac{\omega^{2}+\beta^{2}}{2 \beta}, \quad \mu_{0}=\int_{-\infty}^{\infty} \boldsymbol{\Lambda}(x) d x=\frac{2 c_{0}}{\beta}>1,
\end{gathered}
$$

т.е. $\boldsymbol{\Lambda} \in \Omega_{0}$ является оператором с ядром Лалеско $\boldsymbol{\Lambda}(x)=c_{0} e^{-\beta|x|}$.

Нами доказана

ТЕОРема 4.2. Если в ЗКC, $\mu>1$, ядро $K$ - четная функиия или символ $1-\widehat{K}$ обладает вещественным нулем $\omega>0$, то оператор $\boldsymbol{I}-\boldsymbol{K}$ с ядром (0.2) допускает вещественное разлохение (4.8), әде $\boldsymbol{V}_{ \pm}$определяются, как в теореме 4.1, а $\boldsymbol{\Lambda} \in \Omega_{0}$ оператор с ядром Лалеско, вида (4.9).

4.3. Рассмотрим теперь вопрос о построении двуфакторного разложения оператора $\boldsymbol{I}-\boldsymbol{K}$ в $\mathrm{KC} \mathrm{и} \mathrm{ЗКС.} \mathrm{Из} \mathrm{(4.7)} \mathrm{имеем}$

$$
\boldsymbol{I}-\boldsymbol{K}=\left(\boldsymbol{I}-\boldsymbol{W}_{-}\right)\left(\boldsymbol{I}-\boldsymbol{W}_{+}\right)
$$

где

$$
\boldsymbol{I}-\boldsymbol{W}_{ \pm}=\left(\boldsymbol{I}-\boldsymbol{U}_{ \pm}\right)\left(\boldsymbol{I}-\boldsymbol{V}_{ \pm}\right) .
$$

Ниже мы будем заниматься построением ядер $W_{ \pm}$операторов $\boldsymbol{W}_{ \pm}$. Из $(4.11)$ получаем $\boldsymbol{W}_{ \pm}=\boldsymbol{U}_{ \pm}+\boldsymbol{V}_{ \pm}-\boldsymbol{U}_{ \pm} \boldsymbol{V}_{ \pm}$, откуда

$$
W_{ \pm}(x)=\alpha e^{-\beta x}+V_{ \pm}(x)-\alpha \int_{0}^{x} e^{-\beta(x-t)} V_{ \pm}(t) d t, \quad x>0
$$

Используя (1.12), (4.1) и (3.7), будем иметь

$$
\begin{aligned}
W_{ \pm}(x)=\alpha e^{-\beta x}\left(1-\int_{a}^{b} \frac{p+\beta}{p^{2}+\omega^{2}} \varphi_{ \pm}(p, \beta) d \sigma_{ \pm}(p)\right) & \\
& +\int_{a}^{b} e^{-x p} \varphi_{ \pm}(p, \beta) \frac{p+\beta}{p \pm i \omega} d \sigma_{ \pm}(p) .
\end{aligned}
$$

С учетом (4.3) приходим к следуюшему выражению для $W_{ \pm}$

$$
W_{ \pm}(x)=\int_{a}^{b} e^{-x p} \varphi_{ \pm}(p, \beta)(p+\beta) \frac{p \mp i \omega}{p^{2}+\omega^{2}} d \sigma_{ \pm}(p) .
$$

Покажем, что произведения $(p+\beta) \varphi_{ \pm}(p, \beta)$ не зависят от $\beta \geqslant 0$. Тогда $W_{ \pm}$не зависят от $\beta$. Для этого выпишем УА для $\varphi_{ \pm}(p, 0)$

$$
\varphi_{ \pm}(p, 0)=1+\varphi_{ \pm}(p, 0) \int_{a}^{b} \frac{\varphi_{\mp}(q, 0)}{p+q} \cdot \frac{q^{2}}{q^{2}+\omega^{2}} d \sigma_{\mp}(q) .
$$


Это уравнение соответствует ядру $T=T_{0}$ вида (3.8) при $\beta=0 . \mathrm{B} \S 3$ было отмечено, что это ядро консервативное. Оно также обладает следуюшими легко проверяемыми свойствами:

$$
m_{2}\left(T_{0}\right) \equiv \int_{-\infty}^{\infty} x^{2} T_{0}(x) d x<+\infty, \quad \nu_{1}^{0}=\int_{-\infty}^{\infty} x T_{0}(x) d x=0 .
$$

Первое свойство следует из неравенства

$$
m_{2}\left(T_{0}\right)=2 \sum_{ \pm} \int_{a}^{b} \frac{1}{p\left(p^{2}+\omega^{2}\right)} d \sigma_{ \pm}(p) \leqslant \frac{2}{\omega^{2}} \mu .
$$

Второе из свойств (4.14) является прямым следствием равенства $f_{2}(\omega)=0$ (см. (2.3)). Из утверждения (1.20а)) и (4.14) следует, что

$$
\int_{a}^{b} \frac{\varphi_{ \pm}(p, 0)}{p\left(p^{2}+\omega^{2}\right)} d \sigma_{ \pm}(p)=1
$$

Из конечности второго момента консервативного ядра $T_{0}$ следует (см. [9], [10]) конечность первого момента соответствуюших вольтерровых ядер (1.13), т.е.

$$
\int_{a}^{b} \frac{\varphi_{ \pm}(p, 0) p^{2}}{p^{2}\left(p^{2}+\omega^{2}\right)} d \sigma_{ \pm}(p)<+\infty .
$$

Перепишем (4.13) в виде

$$
\varphi_{ \pm}(p, 0)\left(1-\int_{a}^{b} \frac{\varphi_{\mp}(q, 0)}{p+q} \cdot \frac{q^{2}}{q^{2}+\omega^{2}} d \sigma_{\mp}(q)\right)=1 .
$$

Используя равенства (4.15), получаем

$$
\varphi_{ \pm}(p, 0) \int_{a}^{b} \frac{p}{q(p+q)} \cdot \frac{q^{2} \varphi_{\mp}(q, 0)}{q^{2}+\omega^{2}} d \sigma_{\mp}(q)=1 .
$$

Введем функции

$$
\varphi_{ \pm}^{*}(p, \beta)=\frac{p}{p+\beta} \varphi_{ \pm}(p, 0)
$$

Из (4.17) имеем

$$
\varphi_{ \pm}^{*}(p, \beta) \int_{a}^{b} \frac{p+\beta}{p+q} \cdot \frac{q}{q^{2}+\omega^{2}} \varphi_{\mp}(q, 0) d \sigma_{\mp}(q)=1 .
$$

Заменяя $p+\beta$ на $p+q-(q-\beta)$, имеем

$$
\varphi_{ \pm}^{*}(p, \beta)\left(1-\int_{a}^{b} \frac{q(q-\beta)}{q^{2}+\omega^{2}} \cdot \frac{1}{p+q} \varphi_{\mp}(q, 0) d \sigma_{\mp}(q)\right)=1,
$$

откуда

$$
\varphi_{ \pm}^{*}(p, \beta)\left(1-\int_{a}^{b} \frac{1}{p+q} \varphi_{\mp}^{*}(q, \beta) \frac{q^{2}-\beta^{2}}{q^{2}+\omega^{2}} d \sigma_{\mp}(q)\right)=1 .
$$


Полученное уравнение относительно $\varphi_{ \pm}^{*}(p, \beta)$ совпадает с УА $(1.11),(4.1)$ относительно $\varphi_{ \pm}(p, \beta)$. Из (4.15) и (4.18) следует, что

$$
\int_{a}^{b} \frac{1}{p}\left|\varphi_{ \pm}^{*}(p, \beta)\right| d \rho_{ \pm}(p)=\int_{a}^{b} \frac{1}{p} \varphi_{ \pm}^{*}(p, \beta) \frac{p^{2}-\beta^{2}}{p^{2}+\omega^{2}} d \sigma_{ \pm}(p)<1
$$

В [9], [10] показано, что КР УА (1.11) (при $\lambda<1$ ) является единственным его решением, удовлетворяюшим условию $(4.19)$, поэтому $\varphi_{ \pm}^{*}(p, \beta)=\varphi_{ \pm}(p, \beta)$, т.е.

$$
(p+\beta) \varphi_{ \pm}(p, \beta)=p \varphi_{ \pm}(p, 0)
$$

Нами доказана основная теорема настоящей работы.

TЕОРема 4.3. Пусть в ЗKC, $\mu>1$, ядро $K$ - симметричное или символ $1-\widehat{K}$ обладает вещественным нулем $\omega, \omega>0$. Тогда существует факторизация (4.10), әде $\boldsymbol{W}_{ \pm} \in \Omega_{0}^{+}$. Их ядра $W_{ \pm}$имеют вид (4.12). Они не зависят от выбора допустимого значения $\beta$. Имеет место равенство (4.20), поэто$\mathcal{M} y$

$$
W_{ \pm}(x)=\int_{a}^{b} e^{-x p} \varphi_{ \pm}(p, 0) \frac{1}{p \pm i \omega} d \sigma_{ \pm}(p)
$$

Заметим, что из (4.21) следует, что вешественные части ядер $W_{ \pm}$являются консервативными, имеем

$$
\operatorname{Re} W_{ \pm}(x)=\int_{a}^{b} e^{-x p} \varphi_{ \pm}(p, 0) \frac{p}{p^{2}+\omega^{2}} d \sigma_{ \pm}(p)
$$

поэтому $\operatorname{Re} W_{ \pm} \geqslant 0, \int_{0}^{\infty} \operatorname{Re} W_{ \pm}(x) d x=1$ (см. (4.15)).

Отметим, что преобразование, аналогичное преобразованию (4.20), применяется в теории переноса излучения в рамках метода сдвига Альбедо (см. [19]).

4.4. Результаты настоящего параграфа по факторизации оператора в $\mathrm{KC} \mathrm{и} \mathrm{ЗКС}$ имеют разные сферы применений. Теоремы 4.1-4.3 в ЗКС представляют самостоятельный теоретический интерес как первые результаты по факторизации в ЗКС. На основе построенных факторизаций можно создать достаточно полную конструктивную теорию однородного и неоднородного уравнений $(0.1),(0.2)$ в ЗКС (если символ обладает вещественньм нулем). Часть результатов такого характера будет изложена в $\S 5$. Если однородное уравнение $(0.1),(0.2)$ в ЗКС достаточно подробно исследовано в [12], то результаты по неоднородному уравнению (и подходящие методы) вовсе отсутствовали. Что касается консервативного случая, то построенные в настоящей работе факторизации при $\mu=1$ в основном имеют прикладное значение (в духе метода сдвига Альбедо), способствующие эффективному решению уравнения $(0.1),(0.2)$ в KC, в случае конечности второго момента ядра $K$. Они мало что добавляют к теории консервативных интегральных уравнений Винера-Хопфа, развитой в [9]-[11]. Тем не менее, ниже мы приведем один новый факт по УА в КС, доказываемый с помощью конструкций настоящей работы. 
Лемма 4.2. Пусть в УА (1.11) меры $d \rho_{ \pm}$неотрицательны, удовлетворяют условию консервативности $\lambda=1$ и существует конечный второй момент ядра Т. Тогда КР является единственным решением (1.11), удовлетворяющим условию

$$
\left\|\varphi_{ \pm}\right\|_{\mathscr{L}^{ \pm}} \equiv \int_{a}^{b} \frac{1}{p}\left|\varphi_{ \pm}(p)\right| d \rho_{ \pm}(p) \leqslant 1 .
$$

ДоКАЗАТЕЛЬСТвО. Пусть $\left(\varphi_{+}, \varphi_{-}\right)$удовлетворяет (1.11) и условию (4.22). Применим преобразование типа (4.18), т.е. введем функции

$$
\varphi_{ \pm}^{*}(p, \beta)=\frac{p}{p+\beta} \varphi_{ \pm}(p)
$$

Пара $\left(\varphi_{+}^{*}, \varphi_{-}^{*}\right)$ удовлетворяет уравнению

$$
\varphi_{ \pm}^{*}(p, \beta)=1+\varphi_{ \pm}^{*}(p, \beta) \int_{a}^{b} \frac{\varphi_{\mp}^{*}(q, \beta)}{p+q}\left(1-\frac{\beta^{2}}{q^{2}}\right) d \rho_{\mp}(q) .
$$

В силу конечности $m_{2}(T)$ при достаточно малых положительных значениях $\beta$ вьполняется условие (см. п. 3.2)

$$
\lambda^{*} \equiv \sum_{ \pm} \int_{a}^{b} \frac{1}{q} d \rho_{ \pm}^{*}(q)<1, \quad d \rho_{ \pm}^{*}(q)=\left|1-\frac{\beta^{2}}{q^{2}}\right| d \rho_{ \pm}(q) .
$$

Из оценки (1.15) следует, что

$$
\int_{a}^{b} \frac{1}{p}\left|\varphi_{ \pm}^{*}(p, \beta)\right| d \rho_{ \pm}^{*}(p)<1 \text { при } \lambda^{*}<1 .
$$

Согласно результатам [9], [10] $\left(\varphi_{+}^{*}, \varphi_{-}^{*}\right)$ является единственньм решением (4.23), удовлетворяюшим этому неравенству. Поэтому

$$
\varphi_{ \pm}(p)=\frac{p+\beta}{p} \varphi_{ \pm}^{*}(p, \beta)
$$

единственное решение УА (1.11), удовлетворяюшее условию (4.22). Лемма доказана.

\section{§5. Решение однородного и неоднородного уравнений $(0.1),(0.2)$ в $3 \mathrm{KC}$}

5.1. Настоящий параграф посвящен применению факторизации (4.8) (или (4.7)) к решению однородного и неоднородного уравнений $(0.1),(0.2)$ в случае, когда символ $1-\widehat{K}$ обладает вещественньм нулем. Как уже было отмечено, однородное уравнение достаточно полно исследовано в [12], причем в обшем закритическом случае. Поэтому результатам настояшего параграфа по однородному уравнению мы приписываем в основном методическое значение. Результаты же по неоднородному уравнению мы считаем новыми и конструктивньми. 
Операторы $\boldsymbol{I}-\boldsymbol{V}_{ \pm}$, участвующие в факторизации (4.8), обратимы в пространствах $E^{+}$и в ряде других пространств, в которых мы будем искать решение рассматриваемых уравнений. Поэтому задача в основном сводится к решению однородного и неоднородного уравнений с (симметрическим) закритическим ядром Лалеско

$$
\begin{aligned}
& (\boldsymbol{I}-\boldsymbol{\Lambda}) S_{1}=0, \\
& (\boldsymbol{I}-\boldsymbol{\Lambda}) F_{1}=g_{1},
\end{aligned}
$$

где $\boldsymbol{\Lambda}$ определяется согласно (4.9).

Начнем с изучения (5.1). Имеем

$$
\left(\boldsymbol{I}-\boldsymbol{U}_{-}\right) S_{2}=0
$$

где

$$
S_{2}=\left(\boldsymbol{I}-\boldsymbol{U}_{+}\right) S_{1}
$$

Общее решение (5.3) имеет вид

$$
S_{2}(x)=c e^{i \omega x}, \quad c \in \mathbb{C},
$$

поэтому из (5.4) получаем

$$
S_{1}(x)=c e^{i \omega x}+\alpha c \int_{0}^{x} e^{-i \omega(x-t)} e^{i \omega t} d t .
$$

Итак, однородное уравнение (5.1) имеет вешественное решение

$$
S_{1}(x)=c_{0}(\omega \cos \omega x+\beta \sin \omega x) \equiv A \cos \omega\left(x-x_{0}\right) .
$$

Рассмотрим теперь (5.2). Предположим, что $g_{1}$ - вешественная функция из $L_{1}^{+}$. Пусть комплекснозначная функция $F_{1}$ удовлетворяет уравнению (5.2). Тогда ее вещественная часть $\operatorname{Re} F_{1}$ также будет решением (5.2), а мнимая часть $\operatorname{Im} F_{1}$ будет (тривиальным или нетривиальным) решением однородного уравнения (5.1). Обозначим

$$
\left(\boldsymbol{I}-\boldsymbol{U}_{+}\right) F_{1}=F_{2} .
$$

Имеем

$$
\left(\boldsymbol{I}-\boldsymbol{U}_{-}\right) F_{2}=g_{1} .
$$

Уравнение (5.9) обладает решением вида

$$
F_{2}(x)=g_{1}(x)+g_{2}(x) \equiv g_{1}(x)+\alpha \int_{x}^{\infty} e^{-i \omega(t-x)} g_{1}(t) d t .
$$

Согласно (5.10) функция $F_{2}$ является суммой двух слагаемых, причем первое слагаемое $g_{1}$ из $L_{1}^{+}$, а второе слагаемое $g_{2} \in C_{0}^{+}$. Из (5.8) имеем

$$
F_{1}(x)=F_{2}(x)+\alpha \int_{0}^{x} e^{-i \omega(x-t)} F_{2}(t) d t
$$


откуда и из (5.10) после некоторых выкладок получаем

$$
F_{1}(x)=F_{3}(x)+i\left(c_{1}+\frac{\beta}{\omega} c_{2}\right)(\omega \cos \omega x+\beta \sin \omega x),
$$

где

$$
\begin{gathered}
c_{1}=\int_{0}^{\infty} g_{1}(t) \cos \omega t d t, \quad c_{2}=\int_{0}^{\infty} g_{1}(t) \sin \omega t d t \\
F_{3}(x)=g_{1}(x)-\frac{\beta^{2}+\omega^{2}}{2 \omega} \int_{0}^{\infty} \sin \omega|x-t| g_{1}(t) d t \\
+\left(\frac{\beta^{2}-\omega^{2}}{2 \omega} c_{2}+\beta c_{1}\right) \cos \omega x+\left(\frac{\beta^{2}-\omega^{2}}{2 \omega} c_{1}+\beta c_{2}\right) \sin \omega x
\end{gathered}
$$

Нами построено вешественное решение $F_{3}$ уравнения $(5.2)$ в виде суммы $g_{1}+g_{3}$, где $g_{1} \in L_{1}^{+}, g_{3} \in C_{u}^{+}$.

5.2. Рассмотрим однородное уравнение $(0.1),(0.2)$ при $\mu>1$, т.е.

$$
S(x)=\int_{0}^{\infty} K(x-t) S(t) d t .
$$

Используя факторизацию (4.8), получаем

$$
\left(\boldsymbol{I}-\boldsymbol{V}_{-}\right)(\boldsymbol{I}-\boldsymbol{\Lambda})\left(\boldsymbol{I}-\boldsymbol{V}_{+}\right) S=0 .
$$

Из $\gamma_{-} \equiv\left\|V_{-}\right\|_{L_{1}^{+}}<1$ следует, что уравнение $\left(\boldsymbol{I}-\boldsymbol{V}_{-}\right) S_{2}=0$ имеет только тривиальное решение не только в пространствах $E^{+}$, но и в классах локально интегрируемых на $[0, \infty)$ функций медленного (полиномиального) роста. Поэтому в указанных классах (5.15) эквивалентно уравнению (5.1), где

$$
S_{1}=\left(\boldsymbol{I}-\boldsymbol{V}_{+}\right) S .
$$

Из (5.16) и (5.7) имеем

$$
S(x)=A \cos \omega\left(x-x_{0}\right)+A \int_{0}^{x} \Phi_{+}(x-t) \cos \omega\left(t-x_{0}\right) d t,
$$

где $\Phi_{+} \in L_{1}^{+}$резольвентная функция оператора $\boldsymbol{V}_{+}$, определяемая из (1.3). Поэтому однородное уравнение (5.1) имеет абсолютно непрерывное ограниченное решение (5.17). Этот результат согласуется с результатом [12] по решению однородного уравнения (5.14).

Имеются следующие возможности применения к формуле (5.17) представления вида (1.22) для $\Phi_{ \pm}$. Если $a>0$ и $\beta \leqslant a$, то меры $d \rho_{ \pm}$неотрицательные и представления (1.22) имеют место. Если $a=0$, то можно использовать разложение (3.5) при $\beta=0$. Такой путь близок к подходу работы [12] и имеет тот недостаток, что тогда построение факторизации (1.12) для оператора $\boldsymbol{I}-\boldsymbol{T}$ существенно усложняется. Имеется также другая, нереализованная в настояшей работе, возможность построения представления вида (1.22) в случае знакопеременной меры $\varphi_{ \pm}(p) h(p) d \sigma_{ \pm}(p)$. При этом существенную упрошающую роль сможет играть положительность функций $\varphi_{ \pm}$. Сделанное замечание о представлениях (1.22) относится также к решению неоднородного уравнения (0.1). 
5.3. Рассмотрим неоднородное уравнение $(0.1),(0.2)$ в ЗКС, $\mu>1$, при $g \in L_{1}^{+}$. Введем обозначения

$$
g_{1}=\left(\boldsymbol{I}-\boldsymbol{V}_{-}\right)^{-1} g \in L_{1}^{+}, \quad F_{1}=\left(\boldsymbol{I}-\boldsymbol{V}_{+}\right) f .
$$

В силу факторизации (4.8) $F_{1}$ удовлетворяет уравнению (5.2). Нами уже построено решение (5.13) этого уравнения. Поэтому (0.1) обладает решением

$$
f(x)=F_{1}(x)+\int_{0}^{x} \Phi_{+}(x-t) F_{1}(t) d t .
$$

\section{Нами доказана}

Tеорема 5.1. Пусть в уравнении (0.1), (0.2) $g \in L_{1}^{+}, \mu>1$ и символ $1-\widehat{K}$ обладает вещественным нулем $\omega>0$. Тогда это уравнение имеет решение вида (5.19), где $F_{1}$ определяется из (5.18), а $g_{1} \in L_{1}^{+}$определяется равенством

$$
g_{1}(x)=g(x)+\int_{x}^{\infty} \Phi_{-}(t-x) g(t) d t .
$$

Функиия $f$ является суммой функиий $\left(\boldsymbol{I}+\boldsymbol{\Phi}_{+}\right) g_{1} \in L_{1}^{+}$и $\left(\boldsymbol{I}+\boldsymbol{\Phi}_{+}\right) g_{3} \in C_{u}^{+}$.

\section{§6. Случай комплексных нулей символа}

6.1. В настоящем параграфе мы вкратце рассмотрим вопрос о построении разложений типа (3.1) и (4.7) в случае, когда символ (или его аналитическое продолжение) $1-\widehat{K}$ обладает комплексньми нулями.

Пусть число $s_{1}=\omega+i \xi$ является нулем $1-\widehat{K}$. Тогда согласно $\S 2$ число $s_{2}=-\omega+i \xi$ также является его нулем

$$
1-\widehat{K}\left(s_{m}\right)=0, \quad m=1,2, \quad s_{1,2}= \pm \omega+i \xi, \quad \omega \geqslant 0 .
$$

В случае, когда $s_{1}$ чисто мнимый, т.е. $\omega=0, s_{1,2}=i \xi$. Будем считать, что $s=i \xi$ является нулем второго порядка символа, т.е.

$$
(s-i \xi)^{-2}(1-\widehat{K}(s)) \in C_{0}(-\infty, \infty) .
$$

Пусть $\beta$ - произвольное вешественное число. Из (2.2) и (6.1) получаем

$$
\begin{aligned}
1-\widehat{K}(s) & =\sum_{ \pm} \int_{a}^{b}\left(\frac{1}{p \mp i s_{1}}-\frac{1}{p \mp i s}\right) d \sigma_{ \pm}(p) \\
& =\frac{i s_{1}-i s}{\beta-i s}\left(1-\sum_{ \pm} \int_{a}^{b} \frac{(p \mp \beta)}{(p \mp i s)\left(p \mp i s_{1}\right)} d \sigma_{ \pm}(p)\right),
\end{aligned}
$$

откуда

$$
1-\widehat{K}(s)=\frac{i s_{1}-i s}{\beta-i s}(1-\widehat{G}(s)),
$$

где

$$
G_{ \pm}(x) \equiv G( \pm x)=\int_{a}^{b} e^{-x p} \frac{p \mp \beta}{p \mp i s_{1}} d \sigma_{ \pm}(p)
$$


В силу сделанных предположений о нулях $1-\widehat{K}$ имеем $1-\widehat{G}\left(s_{2}\right)=0$. Поэтому $1-\widehat{G}$ можно разложить аналогично (6.3). При этом число $\beta$ будет заменено на $-\beta$, a $s_{1}$ на $s_{2}$. В результате мы приходим к следуюшему разложению:

$$
1-\widehat{K}(s)=\frac{\left(i s_{1}-i s\right)\left(-i s_{2}+i s\right)}{(\beta-i s)(\beta+i s)}\left(1-\sum_{ \pm} \int_{a}^{b} \frac{\left(p^{2}-\beta^{2}\right)}{(p \mp i s)\left(p \mp i s_{1}\right)\left(p \mp i s_{2}\right)} d \sigma_{ \pm}(p)\right) .
$$

Будем считать, что $\beta>0$. Обозначим

$$
T_{ \pm}(x) \equiv T( \pm x)=\int_{a}^{b} e^{-x p} \frac{p^{2}-\beta^{2}}{(p \pm \xi)^{2}+\omega^{2}} d \sigma_{ \pm}(p) .
$$

Справедливость $T_{ \pm} \in L_{1}^{+}$при $\omega \neq 0$ не вызывает сомнения. При $\omega=0$ это следует из (6.2). Из (6.4) и (6.5) имеем

$$
1-\widehat{K}(s)=\left(1-\widehat{U}_{-}(s)\right)(1-\widehat{T}(s))\left(1-\widehat{U}_{+}(s)\right),
$$

где $1-\widehat{U}_{ \pm}$суть символы операторов $\boldsymbol{I}-\boldsymbol{U}_{ \pm}$:

$$
\begin{aligned}
& \boldsymbol{U}_{+} f(x)=\left(\beta-i s_{1}\right) \int_{0}^{x} e^{-\beta(x-t)} f(t) d t \\
& \boldsymbol{U}_{-} f(x)=\left(\beta+i s_{2}\right) \int_{x}^{\infty} e^{-\beta(t-x)} f(t) d t .
\end{aligned}
$$

Мы пришли к следуюшей лемме.

ЛЕмма 6.1. При выполнении условий (6.1), (6.2) имеет место (6.6) и факторизация

$$
\boldsymbol{I}-\boldsymbol{K}=\left(\boldsymbol{I}-\boldsymbol{U}_{-}\right)(\boldsymbol{I}-\boldsymbol{T})\left(\boldsymbol{I}-\boldsymbol{U}_{+}\right),
$$

где $\boldsymbol{T}$ и $\boldsymbol{U}_{ \pm}$определяются, соответственно, из (6.5) и (6.7).

Аналогично случаю вещественных корней символа, можно показать, что ядро $T$ консервативное в случае $\beta=0$ и выполняется неравенство $\|T\|_{L_{1}(-\infty, \infty)}<1$ при достаточно малых значениях $\beta>0$. Используя эти факты, из (6.8) можно получить разложения типа (4.7), (4.8) и (4.10) в условиях леммы 6.1.

6.2. Сочетая факторизацию (6.8) с теоремой 2.4 об исключительном нуле (см. [12]), можно получить общие результаты по однородному и неоднородному уравнениям $(0.1),(0.2)$ в ЗКС. Мы не излагаем здесь эти факты, поскольку считаем необходимым более подробно исследовать свойства комплексных нулей символа $1-\widehat{K}$ и надеемся найти эффективный алгоритм построения таких нулей.

В работе было построено следующее разложение символа в ЗКС

$$
1-\widehat{K}(s)=\frac{\left(s-s_{1}\right)\left(s-s_{2}\right)}{s^{2}}(1-\widehat{T}(s))
$$

(явный вид $T$ не был найден). Используя это разложение, которое соответствует (6.4) при $\beta=0$, авторами [12] построено решение однородного уравнения (0.1), $(0.2)$ в ЗКС. Разложение (6.9) в [12] не приобрело форму факторизации оператора $\boldsymbol{I}-\boldsymbol{K}$ в винеровской алгебре. Вероятно по этой причине неоднородное уравнение $(0.1),(0.2)$ при $\mu>1$ в [12] не было рассмотрено. 


\section{Список литературы}

1. Амбариумян В. А. Научные труды. Т. І. Ереван, 1960.

2. Чандрасекар C. Перенос лучистой энергии. М.: ИЛ, 1953.

3. Соболев В. В. Перенос лучистой энергии. М.: Гостехиздат, 1956.

4. Busbridge $I$. $W$. The mathematics of the radiative transfer. Oxford, 1960.

5. Черчиньяни $K$. Теория и приложения уравнения Болцмана. М.: Мир, 1978.

6. Енгибарян Н. Б. Об интегралшных уравнениях на полупрямой с разностньми ядрами // Докл. АН Арм. ССР. 1972. Т. 54. № 3. С. 129-133.

7. Енгибарян Н. Б., Арутюнян А. А. Интегральные уравнения на полупрямой с разностным ядром и нелинейные функциональные уравнения // Матем. сб. 1975. Т. 97. № 1. C. $35-58$.

8. Крейн М. Г. О нелинейных интегральных уравнениях, играющих роль в теории уравнений Винера-Хопфа // Матем. исследования. Т. 42. №1. Кишинев: Штиница, 1976. C. $47-90$.

9. Енгибарян Н.Б., Арабаджяя Л. Г. О нелинейных уравнениях факторизации операторов Винера-Хопфо // Препринт ЕГУ. Ереван: НИИ ФКС, 79-1, 1979.

10. Арабаджсян Л. Г., Енгибарян Н. Б. Уравнения в свертках и нелинейные функциональные уравнения // Итоги науки и техники. Математический анализ. Т. 22. М.: ВИНИТИ, 1984. C. $175-244$.

11. Енгибарян Н. Б., Арабаджян Л. Г. Интегральные уравнения Винера-Хопфоа со вполне монотонными ядрами // Изв. АН Арм. ССР. Сер. матем. 1990. Т. 25. № 2. С. 135-155.

12. Крейн М.Г., Шмульян Ю. Л. Уравнения Винера-Хопфа, ядра которых допускают интегральное представление через экспоненты // Изв. АН Арм. ССР. Сер. матем. 1982. T. 17. № 4. С. 307-327; № 5. С. 335-375.

13. Пресдорф З. Некоторые классы сингулярных уравнений. М.: Мир, 1972.

14. Крейн $М . Г$. Интегральные уравнения на полупрямой с ядром, зависящим от разности аргументов // УМН. 1958. Т. 13. №5. С. 3-120.

15. Гохберг И. ЦЦ., Фельдман И. А. Уравнения в свертках и проекционные методы их решения. М.: Наука, 1971.

16. Енгибарян Н. Б., Арабаджсян Л. Г. Некоторыезадачи факторизации для интегральных операторов типа свертки // Дифференц. уравнения. 1990. Т. 26. № 8.

17. Енгибарян Н. Б., Мелконян Э. А. О методе дискретных ординат // ДАН СССР. 1987. T. 292. № 2. C. 322-326.

18. Енгибарян Н. Б., Погосян $A$. А. Об одном классе интегральных уравнений восстановления // Матем. заметки. 1990. Т. 47. №6. С. 23-30.

19. Ivanov V. V., Rybicki G. B., Kasaurov A. M. Albedo Shifting // Preprint Series № 3478. Harvard-Smithsonian Center for Astrophysics, 1992.

Центр математической физики Бюраканской;

Поступила в редакцию Астрофизической обсерватории НАН РА

08.08.1995 